\title{
Can we safely deform a plate to fit every bone? population-based fit assessment and finite element deformation of a distal tibial plate
}

\begin{abstract}
Anatomically precontoured plates are commonly used to treat periarticular fractures. A wellfitting plate can be used as a tool for anatomical reduction of the fractured bone. Recent studies highlighted that some plates fit poorly for many patients due to considerable shape variations between bones of the same anatomical site. While it is impossible to design one shape that fits all, it is also burdensome for the manufacturers and hospitals to produce, store and manage multiple plate shapes without the certainty of utilization by a patient population.

In this study, we investigated the number of shapes required for maximum fit within a given dataset, and if they could be obtained by manually deforming the original plate. A distal medial tibial plate was automatically positioned on 45 individual tibiae, and the optimal deformation was determined iteratively using finite element analysis simulation. Within the studied dataset, we found that: (i) $89 \%$ fit could be achieved with four shapes, (ii) $100 \%$ fit was impossible through mechanical deformation, and (iii) the deformations required to obtain the four plate shapes were safe for the stainless steel plate for further clinical use. The proposed framework is easily transferable to other orthopaedic plates.
\end{abstract}

Keyword: Tibia; Fracture; Implant; Bending; Deformation 3D; Fit Assessment; FEA 